\title{
Prospective Randomized Controlled Study on Treatment of Stage T1-3N0M0 Middle-Lower Section Esophageal Cancer by Two Minimally Invasive Radical Surgeries
}

\author{
Tao Zhang, Hai Jiang* \\ Department of Cardiothoracic Vascular Surgery, Renmin Hospital, Hubei University of Medicine, \\ Shiyan, Hubei, China, 442000 \\ *Corresponding author: hubeiwenbo@qq.com \\ Address: Chaoyang District Road No. 39, Shiyan City, Hubei Province, P.R.China, 442000
}

Keywords: Stage T1-3N0M0; middle-lower section esophageal cancer; Ivor-Lewis surgery; McKeown surgery; prospective study

Abstract: Objective: to conduct contrastive analysis of treatment effect of peritoneoscope plus intrathoracic anastomosis for esophageal cancer resection (Ivor-Lewis surgery) and peritoneoscope plus neck anastomosis for esophageal cancer resection (McKeown surgery) on Stage T1-3N0M0 middle-lower section esophageal cancer. Method: the research period is from January 1, 2015 to December 31, 2017. The research objects include 100 patients with Stage T1-3N0M0 middle-lower section esophageal cancer. They were classified into Ivor-Lewis group (50 cases, treatment with Ivor-Lewis surgery) and McKeown group (50 cases, treatment with McKeown surgery) with random number table. Conventional surgical indexes of patients, surgical index, QLQ-OES18 and postoperative complications were compared and analyzed. Results: the surgery duration, intraoperative blood loss volume, lymph node dissection quantity, and average hospital stay had no significant differences in both groups, and there was no statistical significance (P>0.05). (2) QLQ-C30 3.0 score and QLQ-OES18 score of Ivor-Lewis group were higher than that of McKeown group, and the difference had statistical significance $(\mathrm{P}<0.05)$. (3) The total occurrence rate of complications in Ivor-Lewis group was higher than that of McKeown group, and the difference was significant and had statistical significance $(\mathrm{P}<0.05)$. Conclusion: for treatment of Stage T1-3N0M0 middle-lower section esophageal cancer, Ivor-Lewis surgery and McKeown surgery have little difference in surgical indexes, but complication occurrence rate of Ivor-Lewis group is low, and the living quality is high. Thus, it can be used as the optimal method to treat Stage T1-3N0M0 middle-lower section esophageal cancer.

Surgical excision is often applied for early treatment of esophagus cancer. Although this method has certain therapeutic effect, postoperative recovery of patients is slow and there are multiple complications. Meanwhile, postoperative living quality of patients is $l o w^{[1]}$. The development of minimally invasive technology provides more methods for esophagus cancer treatment. Compared with surgical excision, obvious minimally invasive advantage exists. In recent years, some 
literatures indicate that Ivor-Lewis surgery is more preponderant than McKeown surgery in treatment of middle-lower section esophageal cancer, which is mainly reflected in complication occurrence aspect ${ }^{[2]}$. Considering the above research belongs to retrospective analysis report, prospective randomized controlled study will be applied in this paper to discuss the application of Ivor-Lewis surgery and McKeown surgery in Stage T1-3N0M0 middle-lower section esophageal cancer. The detailed analysis is as follows:

\section{Clinical Data and Method}

\subsection{General data}

The research period is from January 1, 2015 to December 31, 2017. The research objects include 100 patients with Stage T1-3N0M0 middle-lower section esophageal cancer. They were classified into Ivor-Lewis group and McKeown group with random number table. Ivor-Lewis group: 28 male patients, 22 female patients, age 38-77, average age 55.8 \pm 3.9 . McKeown group: 27 male patients, 23 female patients, age 36-76, average age 55.6 \pm 4.1 . The above data of patients in both groups conform to comparability requirement $(\mathrm{P}>0.05)$.

\subsection{Inclusion and exclusion criteria}

Inclusion criteria: (1) patients with Stage T1-3N0M0; (2) the focus is in the middle chest or lower chest section below the knuckle; (3) not receive preoperative neoadjuvant chemoradiotherapy; (4) received mechanical anastomosis of tube-type anastomat; (5) the research scheme is approved by the hospital ethics committee, and patients and their relatives sign the informed consent. Exclusion criteria: (1) cervical or thoracic operation history; (2) combined pulmonary interstitial fibrosis, asthma, chronic obstructive pulmonary disease and diabetes; (3) combined heart, brain, liver and kidney dysfunction; (4) other malignant tumors; (5) hematological system diseases; (6) spirit system disease and incomplete research data.

\subsection{Method}

Ivor-Lewis group: general anesthesia; supine position; established artificial pneumoperitoneum; put one $5 \mathrm{~mm}$ puncture outfit below the xiphoid for pulling the liver; conducted the dissection of lymph gland beside the left artery, coeliac trunk and common hepatic artery; dissociated and arrived at the adipose tissue on the side of greater curvature; completely dissociated gastric body; the ultrasound knife dissociated adipose and lymph gland tissue on the side of lesser curvature to complete enterocoelia operation; later, left lateral position; established 3 incisions; dissociated the tumor and conducted the dissection of mediastinal lymph node; mechanical anastomosis of esophagus-stomach tube; manually sutured the esophagus near the top chest; completed esophagus-stomach end-to-side anastomosis for the right chest; sealed lesser curvature tissue and incision with the staple cartridge; sutured and fixed the entire layer of the stomach tube incision $\operatorname{margin}^{[3]}$.

McKeown group: general anesthesia; left lateral position; established 3 handling holes; the endoscope entered the chest for dissection of lymph gland beside the right recurrent laryngeal nerve; dissociated thoracic esophageal; completed double ligation behind the arch of azygos vein; cut off with the ultrasound knife; dissociated the top of thoracic esophagus to diaphragm gap; after the completion of esophagus dissociation, conducted mediastinal lymph node dissection; adjusted to the supine position; abdominal surgery operation was basically consistent with Ivor-Lewis surgery ${ }^{[4]}$. 


\subsection{Observation indexes}

Observation indexes: (1) surgical indexes: (2) recent living quality; (3) postoperative complication occurrence.

\subsection{Evaluation indexes}

Postoperative living quality of patients was scored with Quality Life Questionnaire of European Organization for Research and Treatment of Cancer (QLQ-C30 3.0) and evaluated with supplementary scale for esophagus cancer (QLQ-OES18) ${ }^{[5]}$.

\subsection{Statistical analysis}

SPSS21.0 software was used for data analysis. Surgical indexes and living quality scores were expressed as mean \pm standard deviation, and tested with $t$ test. The complication occurrence was expressed with \%, and the difference was tested with chi-square test. Statistical significance standard: $\mathrm{P}<0.05$.

\section{Results}

\subsection{Comparison of surgical indexes}

The surgery duration, intraoperative blood loss volume, lymph node dissection quantity, and average hospital stay had no significant differences in both groups, and there was no statistical significance $(\mathrm{P}>0.05)$.

\subsection{Life quality score}

Based on the comparison of scores of various items in QLQ-C30 3.0 and QLQ-OES18, Ivor-Lewis group was higher than McKeown group, and the difference had statistical significance $(\mathrm{P}<0.05)$.

\subsection{Comparison of complication occurrence}

In terms of such complications as anastomotic fistula, anastomotic stenosis, and recurrent laryngeal nerve injury, Ivor-Lewis group and McKeown group had large differences and there was statistical significance $(\mathrm{P}<0.05)$. But in the aspects of chylothorax, arrhythmia and gastric emptying, the difference between the observation group and control group had no statistical significance $(\mathrm{P}>0.05)$, as shown in Tab.1.

Tab.1 Comparison of complication occurrence after treatment [n(\%)n=50]

\begin{tabular}{cccccccc}
\hline Group & $\begin{array}{c}\text { Anastomoti } \\
\text { c fistula }\end{array}$ & $\begin{array}{c}\text { Anastomotic } \\
\text { stenosis }\end{array}$ & $\begin{array}{c}\text { Recurrent } \\
\text { laryngeal } \\
\text { nerve injury }\end{array}$ & Chylothorax & Arrhythmia & $\begin{array}{c}\text { Gastric } \\
\text { emptying }\end{array}$ & Total \\
\hline $\begin{array}{c}\text { Ivor-Lewis } \\
\text { group }\end{array}$ & $1(2.00)$ & $1(2.00)$ & $1(2.00)$ & $3(6.00)$ & $4(8.00)$ & $3(6.00)$ & $13(26.00)$ \\
$\begin{array}{c}\text { McKeown } \\
\text { group }\end{array}$ & $7(14.00)$ & $7(14.00)$ & $7(14.00)$ & $4(8.00)$ & $5(10.00)$ & $4(8.00)$ & $34(68.0)$ \\
$\chi^{2}$ & 4.891 & 4.891 & 4.891 & 0.153 & 0.122 & 0.153 & 17.703 \\
$P$ & 0.026 & 0.026 & 0.026 & 0.695 & 0.726 & 0.695 & 0.000 \\
\hline
\end{tabular}




\section{Discussion}

Based on retrospective analysis and research, the application of Ivor-Lewis surgery and McKeown surgery in treatment of Stage T1-3N0M0 middle-lower section esophageal cancer was analyzed through prospective study. The results showed that, both methods conform to the advantages of minimally invasive therapy, and the differences in surgical indexes were small. In the aspect of postoperative life quality score, the comparison between the scores of various items in QLQ-C30 3.0 and QLQ-OES18 indicated that, Ivor-Lewis group was higher than McKeown group. This means that, Ivor-Lewis surgery contributes more to improving patients' living quality after the operation. The complication occurrence rate of Ivor-Lewis group (26.00\%) was lower than that of McKeown group (68.0\%). This indicates that, Ivor-Lewis surgery has higher safety. This may be because the living quality of patients in this group is higher after the operation ${ }^{[6-9]}$. Clinical literatures and reports indicate that, Ivor-Lewis surgery is relatively simple during the treatment of middle-lower section esophageal cancer, while McKeown surgery is relatively complex and increases the risk of recurrent laryngeal nerve injury, thus reducing patients' living quality. This conclusion is consistent with the research result of this paper ${ }^{[10]}$.

In conclusion, Ivor-Lewis surgery and McKeown surgery have small differences in surgical indexes during the treatment of Stage T1-3N0M0 middle-lower section esophageal cancer. But, the postoperative complication occurrence rate of Ivor-Lewis surgery is low, and the living quality is higher. Therefore, Ivor-Lewis surgery is the first choice for the treatment of Stage T1-3N0M0 middle-lower section esophageal cancer.

\section{References}

[1] Chunbo Zhai, Yongjing Liu, Wei Li.A comparison of short-term outcomes between Ivor-Lewis and McKeown minimally invasiveesophagectomy.J Thorac Dis. 2015 Dec; 7(12): 2352-2358.

[2] Ju-Wei Mu, Shu-Geng Gao, Qi Xue.Updated experiences with minimally invasive McKeown esophagectomy for esophageal cancer.World J Gastroenterol. 2015 Dec 7; 21(45): 12873-12881.

[3] Baofu Chen, Bo Zhang, Chengchu Zhu,Modified McKeown Minimally Invasive Esophagectomy for Esophageal Cancer: A 5-Year Retrospective Study of 142 Patients in a Single Institution.PLoS One. 2013; 8(12): e82428.

[4] Ju Sik Yun, Kook Joo Na, Sang Yun Song,Comparison of perioperative outcomes following hybrid minimally invasive versus open Ivor Lewisesophagectomy for esophageal cancer.J Thorac Dis. 2017 Sep; 9(9): 3097-3104.

[5] Frans van Workum, Gijs H. Berkelmans, Bastiaan R.McKeown or Ivor Lewis totally minimally invasive esophagectomy for cancer of the esophagus and gastroesophageal junction: systematic review and meta-analysisJ Thorac Dis. 2017 Jul; 9(Suppl 8): S826-S833.

[6] Babatunde A. Yerokun, Zhifei Sun, Minimally Invasive Versus Open Esophagectomy for Esophageal Cancer: A Population-Based Analysis .Ann Thorac Surg. 2016 Aug; 102(2): 416-423.

[7] Lei Chen, Xi Liu, Rong Wang, Yuncang Wang, Minimally invasive esophagectomy for esophageal cancer according to the location of the tumor: Experience of 251 patients.Ann Med Surg (Lond) 2017 May; 17: 54-60.

[8] Grzegorz Wallner, Witold Zgodziński, Wioletta Masiak-Segit,Minimally invasive surgery for esophageal cancerbenefits and controversies.Kardiochir Torakochirurgia Pol. 2014 Jun; 11(2): 151-155.

[9] Magnus Nilsson, Satoshi Kamiya, Mats Lindblad, Ioannis Rouvelas.Implementation of minimally invasive esophagectomy in a tertiary referral center for esophageal cancer.J Thorac Dis. 2017 Jul; 9(Suppl 8): S817-S825.

[10] K. W. Maas, S. S. A. Y. Biere, J. J. G. Scheepers, S. S. Gisbertz, V. Turrado Rodriguez, D. L. van der Peet, M. A. Cuesta.Minimally invasive intrathoracic anastomosis after Ivor Lewis esophagectomy for cancer: a review of transoral or transthoracic use of staplers.Surg Endosc. 2012 Jul; 26(7): 1795-1802. 